\title{
Design of a Linear Electrical Machine for a Wave Generation System in the Maltese Waters
}

\author{
Xuereb Annalise ${ }^{1}$, Spiteri Staines Cyril $^{1}$, Sant Tonio ${ }^{1}$, Mule`Stagno Luciano ${ }^{2}$ \\ ${ }^{1}$ Faculty of Engineering ${ }^{2}$ Institute of Sustainable Energy \\ University of Malta, Msida, MSD2080, Malta \\ Tel: (+356) 23402340, Fax: (+356) 23402342 \\ Email:*annalisexuereb@gmail.com; cyril.spiteri-staines@um.edu.mt \\ tonio.sant@um.edu.mt; luciano.mule-stagno@um.edu.mt
}

Topic: Ocean Energy

Keywords: wave energy converter, linear generator, tubular machine, ANSYS Maxwell

\begin{abstract}
This research concerns the design of an electrical machine that shall be implemented on a wave energy converter (WEC). Analysis of the available wave data and optimisation of the WEC were carried out so as to design the optimum system that will interact efficiently with the conditions of the selected location. The electrical model was designed for wave heights and periods that yielded the maximum power throughout the year. The initial finite element simulations were undertaken on the design of a 4-pole tubular permanent magnet (PM) linear synchronous machine. The performance of the system was optimised for a wave height and period of 3 meters and 6 seconds, respectively. The capacity of the linear generator was set at 15kVA and the dimensions of the WEC (floating point absorber) were determined accordingly through the application of a simplified model for predicting the coupled dynamic response of the absorber and the linear generator. It was necessary that the machine gives a reasonable performan+ce when operating under the slower and most common waves. Thus an 8-pole and a 16-pole tubular linear PM machine were also simulated and their performance was compared. Through this analysis the 16-pole design resulted in the best performance, especially at low speeds. Simulations were initially compared at fixed speed but were later simulated with variable speed conditions so as to represent more accurately the wave's motion and monitor the power generator performance at variable loadings.
\end{abstract}

\section{Introduction}

Utilisation of renewable resources of energy is constantly becoming a necessity in the power generation industry. This led to the design of new concepts while improving those which are already in operation. Wave energy is one important source of renewable energy for small countries like Malta which have severe spatial restrictions onshore due to a high population density but on the other hand have a large accessibility to the coastline.

The design of a wave energy converter is driven by the need to achieve efficient operation in the local conditions. Thus analysis of the actual wave power data was an important step in the machine design optimization process. In the present study, wave data was gathered through instrumentation located on a floating buoy that was anchored at a selected location in Maltese waters. Analsyis of the measurement data confirmed that wave conditions are characterized by low frequency waves with relatively small amplitudes. This indicated that a point absorber converter would be a suitable concept[1]. The study considered a point absorber converter coupled to a linear electrical generator as a power take-off system to generate electricity from vertical oscillations imparted by wave motion.

Different linear machine configurations were investigated, the ultimate aim being that of maximising the amount of power extracted at relatively low speeds as the selected locations. A design concept involving a toothed tubular linear machine was selected. The latter was designed with a fixed armature and a moving magnetic translator. The linear generator design was optimised through simulations that were carried out in ANSYS Maxwell[2]. ANSYS Maxwell is a finite element modelling software for the design and analysis of electromagnetic fields and electromechanical devices. Further simulations were also carried out on ANSYS Simplorer [3]so as to monitor the electrical characteristics of the machine. 


\section{Analysis of Local Wave Power Data}

Wave monitoring at the location chosen for implementation was an important step in the system's design. Data was gathered through wave measuring instruments enclosed in a floating buoy anchored on the North West side of Gozo (the most northern island of the Maltese Islands) close to $2 \mathrm{~km}$ away from land at a depth of 200m. Due to its rounded shape, the direction of the wave front does not influence the buoy's motion and the buoy oscillated vertically on the wave's surface. The location selected is far away from land or any other floating structure. Thus the effects of diffraction, reflection and radiation resulting from coastal features or other buoyant structures could be assumed to by minimal.

The wave data was collected every 30 minutes and spanned over a year from the beginning of October 2011 till the end of September 2012. The measurements and subsequent analysis were conducted by the International Oceanographic Institute of the University of Malta. The analysis showed that the lowest wave heights were about $10 \mathrm{~cm}$ and had a corresponding period of $2.26 \mathrm{~s}$. As expected, an increase in wave height brings with it an increase in wave period and the buoy even experienced an extreme $7.5 \mathrm{~m}$ waves with a period of $8.9 \mathrm{~s}$. The most common waves at the wave buoy station had a time period between 3 and 3.5 seconds and a wave height that varies between 0.2 and 0.4 meters. Further calculations also showed a peak vertical velocity for the recorded waves in the range $0.14 \mathrm{~m} / \mathrm{s}$ and $2.65 \mathrm{~m} / \mathrm{s}$. However power is higher for waves with larger wave heights and periods and consequently waves with a lower frequency of occurence may result in a larger quantity of energy being generated over a period of time. Thus to examine which waves are in possession of the highest energy potential throughout the year, wave data was also analysed in terms of its probability of occurrence across the recorded period. Figure 1 shows that the most common waves did not result in the highest power distributed throughout the recorded year. These calculations show that the waves which yielded most energy over the recorded year have a period between 5.5 and 6 seconds, a wave height varying between 2.8 and 3 meters and a peak vertical velocity reaching $1.57 \mathrm{~m} / \mathrm{s}$.

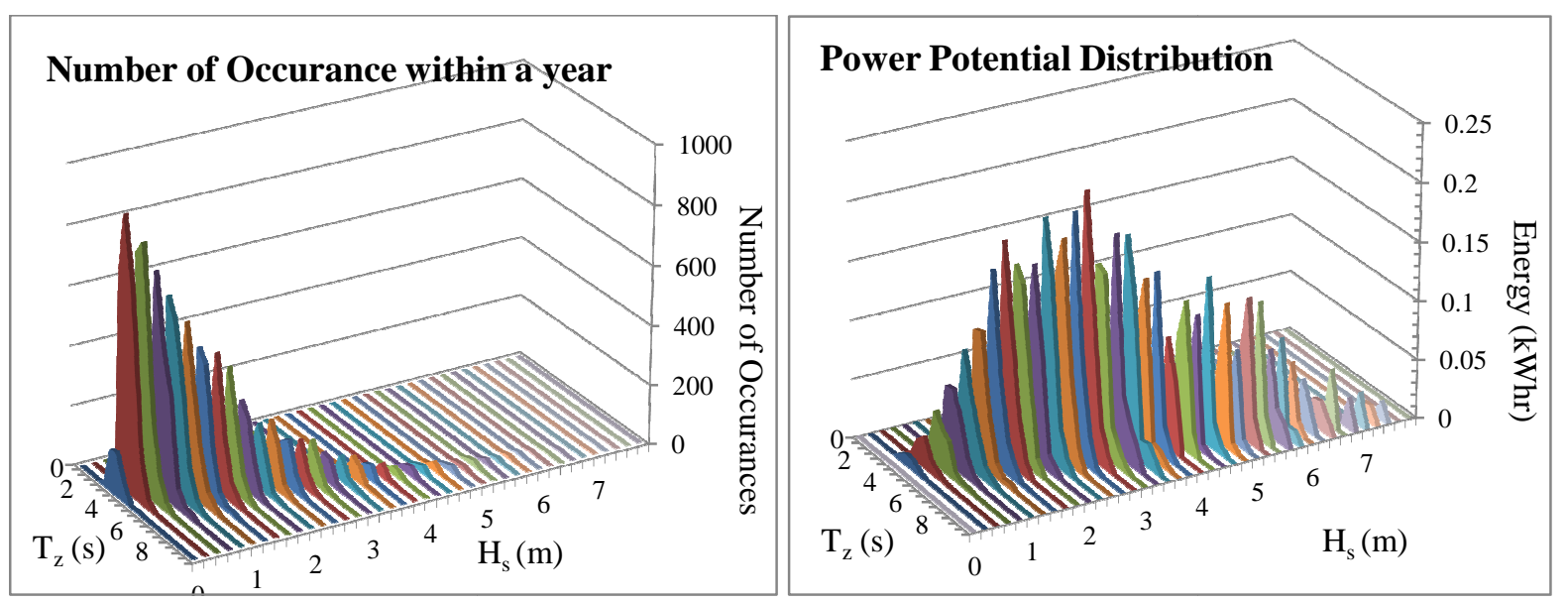

Figure 1: Number of wave occurrences and power potential distributions of waves in a year for different wave height $\boldsymbol{H}_{\boldsymbol{s}}(\mathrm{m})$ and zero up-crossing period $\boldsymbol{T}_{\boldsymbol{z}}(\mathrm{s})$

\section{Dynamic Modelling of the WEC}

The aim of this project was to develop the design of a linear electrical generator for a direct drive WEC system. Due to the wide range of wave speeds at the selected locations the linear generator being proposed was coupled to a point absorber converter. Point absorbers are floating devices that can either be semi-submerged or fully-submerged. These converters are anchored to the seabed and operate by varying their displacement from the mean level of the water's surface with every crest or trough [4].

The dynamic response of a point absorber system is modelled by a simple mass-spring-damper-system having a single degree of freedom. The system consists of a floating buoy coupled to a PTO system as shown in Figure 2 . The proposed design concept includes a linear generator, which is modelled by a damper and anchored to the seabed 
through additional retracting mechanical springs. These springs are used so as to maintain the link between the floating buoy and the translator in tension.

In the present analysis a single floating mass was assumed to be anchored to the sea bed through a PTO system. During the analysis it was also assumed that the WEC is subjected to single frequency waves and that the floating mass is allowed to move only in heave mode. Thus only the vertical components of the forces acting on the system were considered. It was also assumed that the wave's amplitudes and motions are relatively small for the governing equations to work out. Using these assumptions the response may then be also analysed in the frequency domain [5]. In practice there will exist both vertical and horizontal components which require a more complex mechanical system model. It was also assumed that the buoy will be large enough to be able to pull the translator's weight upwards and will be continuously floating at the sea water surface, unless the wave height is beyond the allowable displacement of the linear generator.

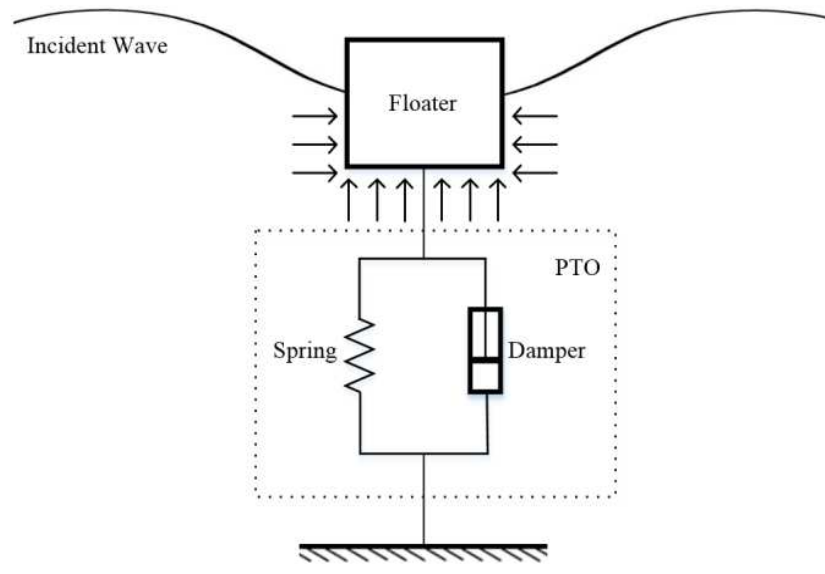

Figure 2: Point absorber model with the linear generator represented by a damper

With these assumptions in mind the linear generator was therefore assumed to follow a vertical motion. The linear machine design shall thus be based on a sinusoidal wave motion with a maximum vertical velocity of $1.57 \mathrm{~m} / \mathrm{s}$ for the $3 \mathrm{~m}$ and $6 \mathrm{~s}$ waves selected. Following this analysis the final generator design shall also be tested for variable speed conditions.

The heaving force of the incident wave $f_{d}$ can be represented as [5]

$$
f_{d}=\left(m+m_{a}\right) \ddot{x}+\left(b_{\text {hyd }}+D_{\text {pto }}\right) \dot{x}+\left(K_{\text {pto }}+\rho g A_{0}\right) x
$$

where $m$ is the mass of floating buoy; $m_{a}$ is the hydrodynamic coefficient of added mass to cater for the effect of the inertia of the water on the floater; $b_{\text {hyd }}$ is the hydrodynamic damping coefficient to account for the damping of the floating buoy when energy is transferred through the radiated waves; $D_{\text {pto }}$ is the damping coefficient of the linear damper; $K_{\text {pto }}$ is the linear spring stiffness constant; $\rho$ is the sea water density; $g$ is the acceleration due to gravity; $A_{0}$ is the cross sectional area of the floater and $\mathrm{x}, \dot{\mathrm{x}}$ andẍare the position, the vertical speed and vertical acceleration of the buoy.

Equation (1) shows that the behaviour of a heaving point absorber converter can be modelled by a second order expression. Such systems achieve their optimum performance when operating at the resonant frequency $\omega_{n}$. The natural frequency of the complete WEC is equal to:

$$
\omega_{n}=\sqrt{\frac{\rho g A_{0}+K_{p t o}}{m+m_{a}}}
$$


Equation (1) may be expressed in the s-domain in terms of $X_{o}(s)$ as

$$
X_{o}(s)=\frac{F_{d}}{s^{2}\left(m+m_{a}\right)+s\left(b_{\text {hyd }}+D_{p t o}\right)+\left(K_{p t o}+\rho g A_{0}\right)}
$$

The energy absorbed by the PTO system is mainly due to the damping component. The stiffness of the PTO system $K_{\text {pto }}$ does not contribute to the effective power absorbed by the electrical machine. This leads to a time-averaged power absorbed of:

$$
\bar{P}_{e}=\frac{\Delta W}{T}=\frac{\pi D_{p t o} \omega\left|X_{o}\right|^{2}}{\frac{2 \pi}{\omega}}=\frac{1}{2} D_{p t o} \omega^{2}\left|X_{o}\right|^{2}
$$

Thus Equation (4) shows that the power is dependent on motion's frequency $\omega$ and the maximum displacement $X_{0}$. Besides that, in [5], the author also shows that for maximum power absorption, apart from operating at the resonant frequency $\omega_{n}$, the hydrodynamic constant $b_{\text {hyd }}$ needs to be equal to the PTO damping $D_{p t o}$.

The dynamic response of a WEC system was analysed in detail and optimisation of the floating buoy was carried out in WAMIT; a commercial code to simulate hydrodynamic loads on offshore structures, not only WECs. This analysis was possible through the invaluable support of the research institute in WavEC Offshore Renewables in Portugal. Simulations were carried out on a $1 \mathrm{~m}$ and $4 \mathrm{~m}$ draft buoys, which were expected to reach their resonance frequency at $2 \mathrm{rad} / \mathrm{s}(3.1 \mathrm{~s}$ wave) and $1.5 \mathrm{rad} / \mathrm{s}(4.2 \mathrm{~s}$ wave) respectively. The shorter buoys were able to absorb power from slower moving waves but a larger range of waves was absorbed by the $1 \mathrm{~m}$ draft buoy.

In[5], Falcão shows that for maximum power absorption the damping of the floating system needs to be equal to the damping created by the linear machine. The latter was calculated using the machine's force and velocity parameters and a $2 \mathrm{~m}$ radius buoy with a draft of $1 \mathrm{~m}$ was selected. It was also required to ensure that the selected buoy will float when selecting the mass required for correct operation with the PTO system at $1.05 \mathrm{rad} / \mathrm{s}$. Since a $2 \mathrm{~m}$ radius would not be able to accommodate the mass calculated, a $4 \mathrm{~m}$ radius buoy was considered. Consequently this wider buoy obtained a larger hydrodynamic damping effect than that obtained for the linear generator. Thus the effect of different values of PTO damping of the $4 \mathrm{~m}$ radius buoy was analysed through the equation of motion. It could be noted that for low values of PTO damping the peak response is closer to $2 \mathrm{rad} / \mathrm{s}$ however a system with higher values of PTO damping exhibit a wider-banded wave energy absorption characteristic which allow for correct operation even with the linear generator selected. More details on the above results can be found in [6].

\section{Linear Generator Design}

The tubular linear permanent magnet (PM) synchronous machine model was initially based on a design reported in [7] with a fixed armature and a moving magnetic translator. Ring magnets magnetized parallel to the direction of motion were mounted in alternating directions on an aluminium shaft to form the translator. Ring phase windings make up the stator of the tubular design and are enclosed in steel covers and separated by aluminium rings as in Figure 3 and Figure 4.

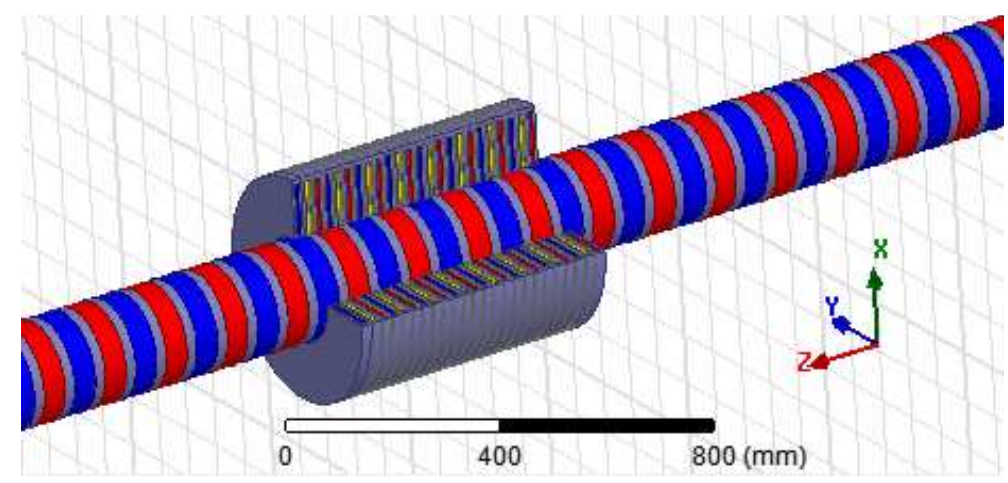

Figure 3: 3D view of the 16-pole tubular linear machine 
Simulations were carried out in ANSYS Maxwell. The model was initially simulated through the Transient solver and later coupled to the electrical loading circuitry in Simplorer. In Simplorer the model is coupled to a star connected resistive load of $22 \Omega$ per phase and the machine is operated at a speed of $1.57 \mathrm{~m} / \mathrm{s}$. The simulation schematic is shown in Figure 5.

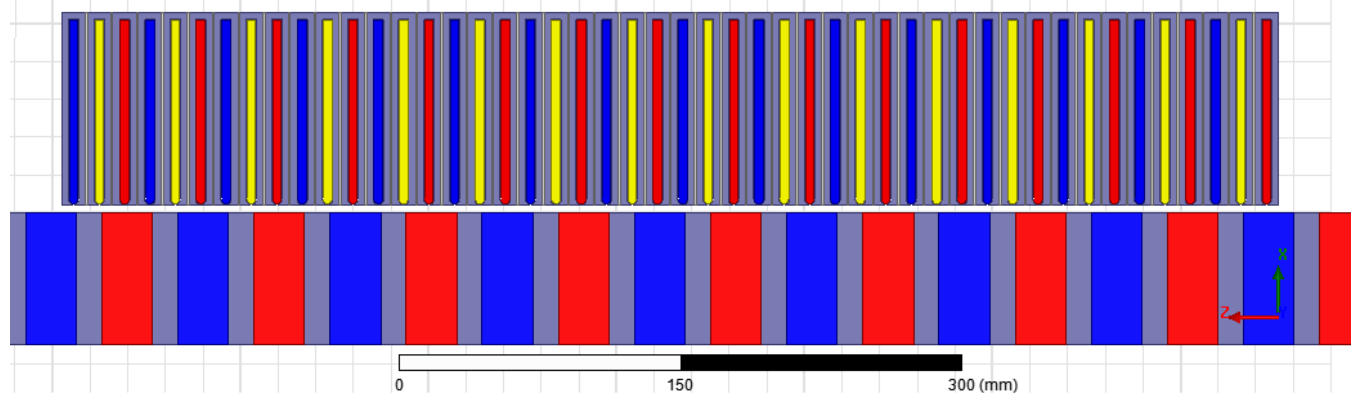

Figure 4: Sectional view of the 16-pole tubular linear machine

Initial simulations were carried out on a 4-pole configuration to optimise a $15 \mathrm{kVA}$ machine at $1.57 \mathrm{~m} / \mathrm{s}$. However the electrical outputs of this machine at the required speeds had a very low electrical frequency and a very distorted output. Thus simulations on models with shorter pitched translators were carried out. To keep the 4-pole configuration the width of each armature segment was reduced with a similar factor and in order to maintain a similar performance the total number of winding turns per phase must remain constant. Consequently the coils need to be thinner for shorter pitched machines, but longer to fit in the same number of coils.

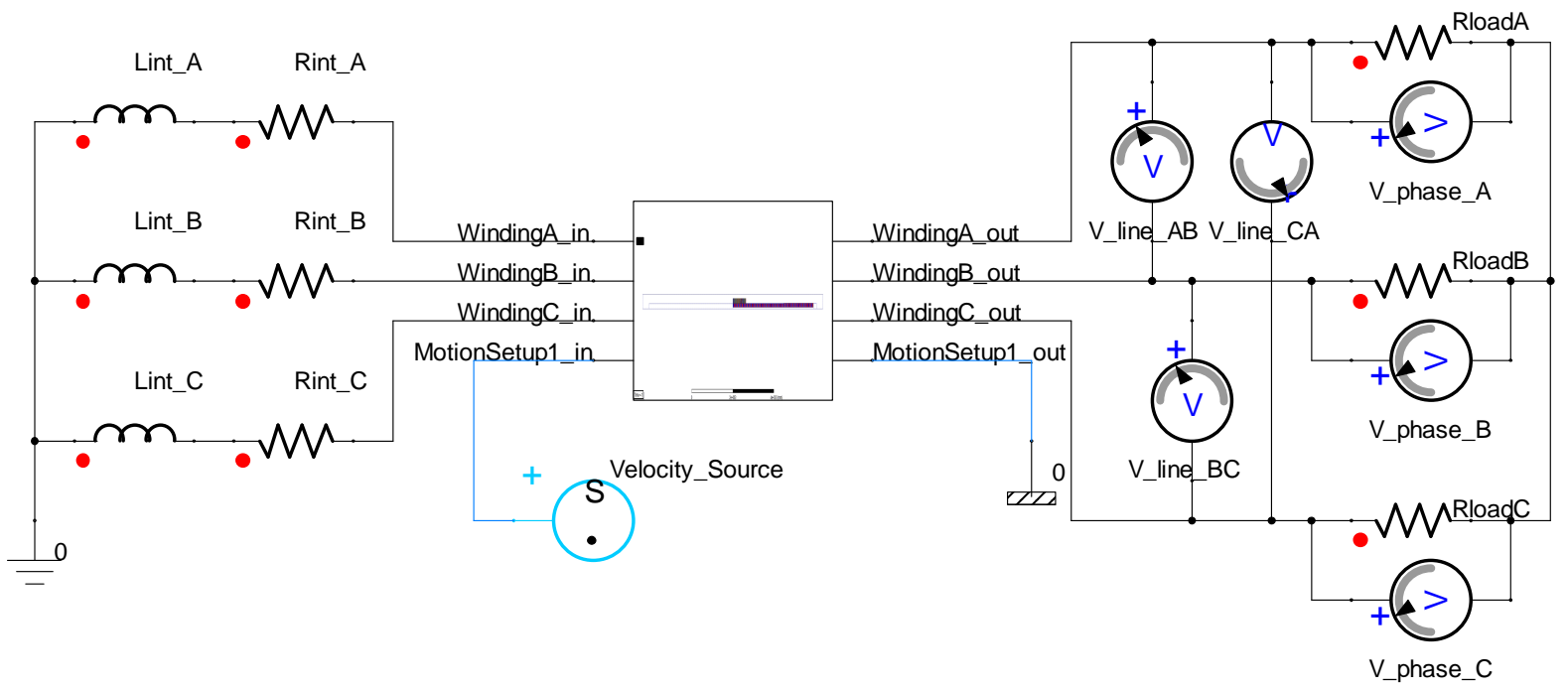

Figure 5: Maxwell model block coupled to the electrical components in Simplorer

Thus the number of turns per coils was subdivided into different stator segments so as to model an 8- and a 16-pole linear machine which are equivalent to the 4-pole design. Attention was given to the saturation levels in the stator which led to the modification of the stator teeth. Thus the teeth are modelled with a semi-open configuration so as to allow the flux lines to flow through a wider path and reduce the saturation stresses on the teeth edges.

Simulations comparing the different configurations showed that the translator of the larger pole configuration exerted a smoother force and managed to achieve a smoother voltage with an increase in the electrical frequency for the same velocity of $1.57 \mathrm{~m} / \mathrm{s}$, as shown in Figure 6 .

It was also important to analyse how the linear generator would perform at variable speed conditions. Thus a sinusoidal velocity input was prescribed to the model block in Figure 5 with a peak velocity of $1.57 \mathrm{~m} / \mathrm{s}$ and a period of $6 \mathrm{~s}$ (the period of the selected waves for which the linear generator is being designed). Under these conditions the 
translator will move a peak to peak distance of $3 \mathrm{~m}$. For a continuous output from all the coils on the stator the length of the translator needs to be longer than the sum of the distance that the translator will move and the length of the stator. Thus the PM machine was designed with a translator of $4 \mathrm{~m}$ length for a stator of $0.7 \mathrm{~m}$ length and an outer radius of $0.9 \mathrm{~m}$. However since there exists the possibility that the wave displacement can even exceed this length, a mechanical braking system needs to be incorporated in the WEC to limit its oscillations when the buoy is hit by large wave heights. Waves recorded with a displacement close to $3.5 \mathrm{~m}$ had a peak velocity of $1.83 \mathrm{~m} / \mathrm{s}$.
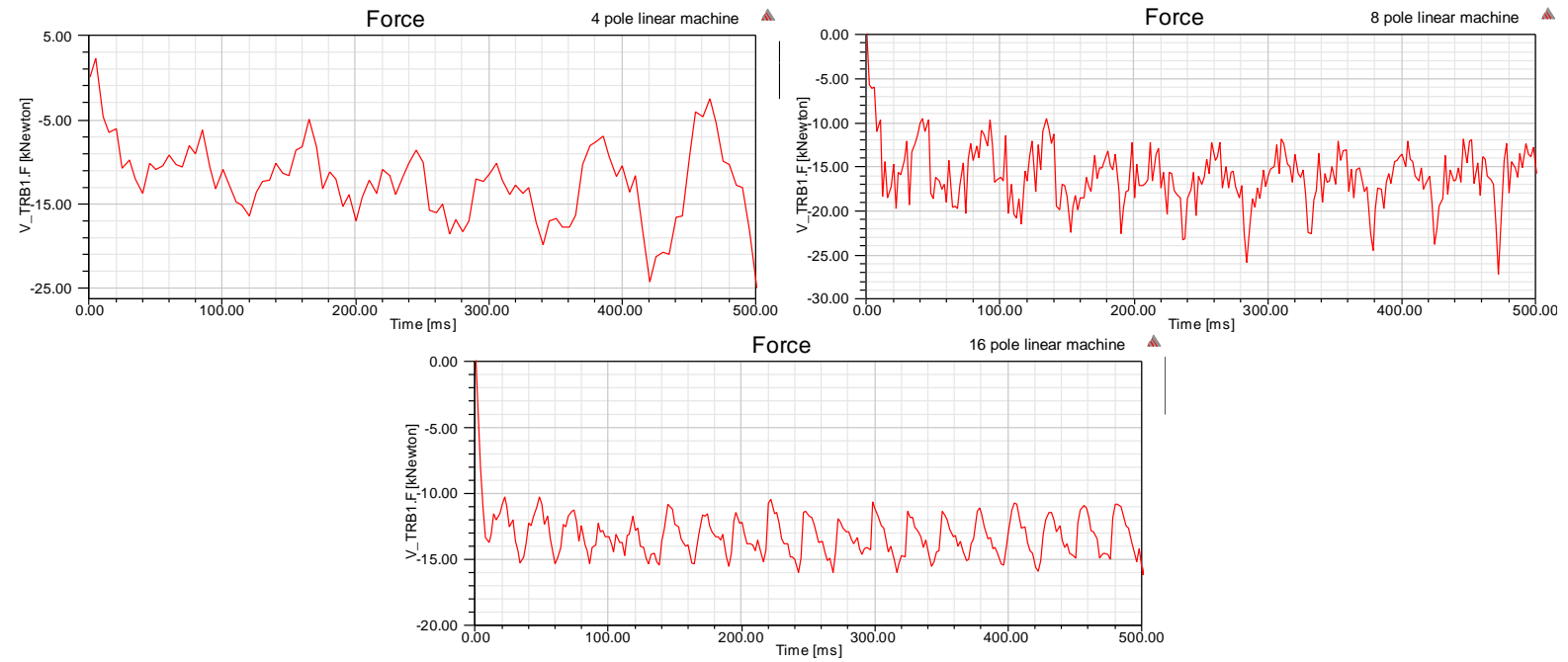

Figure 6: The force exerted on the translator of the 4-, 8-, 16- pole machines

Energy in larger waves can also be absorbed if a longer translator is used. Slower conditions were also analysed and the speed of $0.359 \mathrm{~m} / \mathrm{s}$ was selected to represent waves with a period of $3.4 \mathrm{~s}$ and a maximum wave height of $0.6 \mathrm{~m}$. This condition was selected based on the most common type of waves recorded. Simulations of the three linear machines were carried out with maximum speeds of $1.83 \mathrm{~m} / \mathrm{s}$ (the highest speed allowed by the $4 \mathrm{~m}$ translator system) and $0.359 \mathrm{~m} / \mathrm{s}$ (speed of the most common waves).

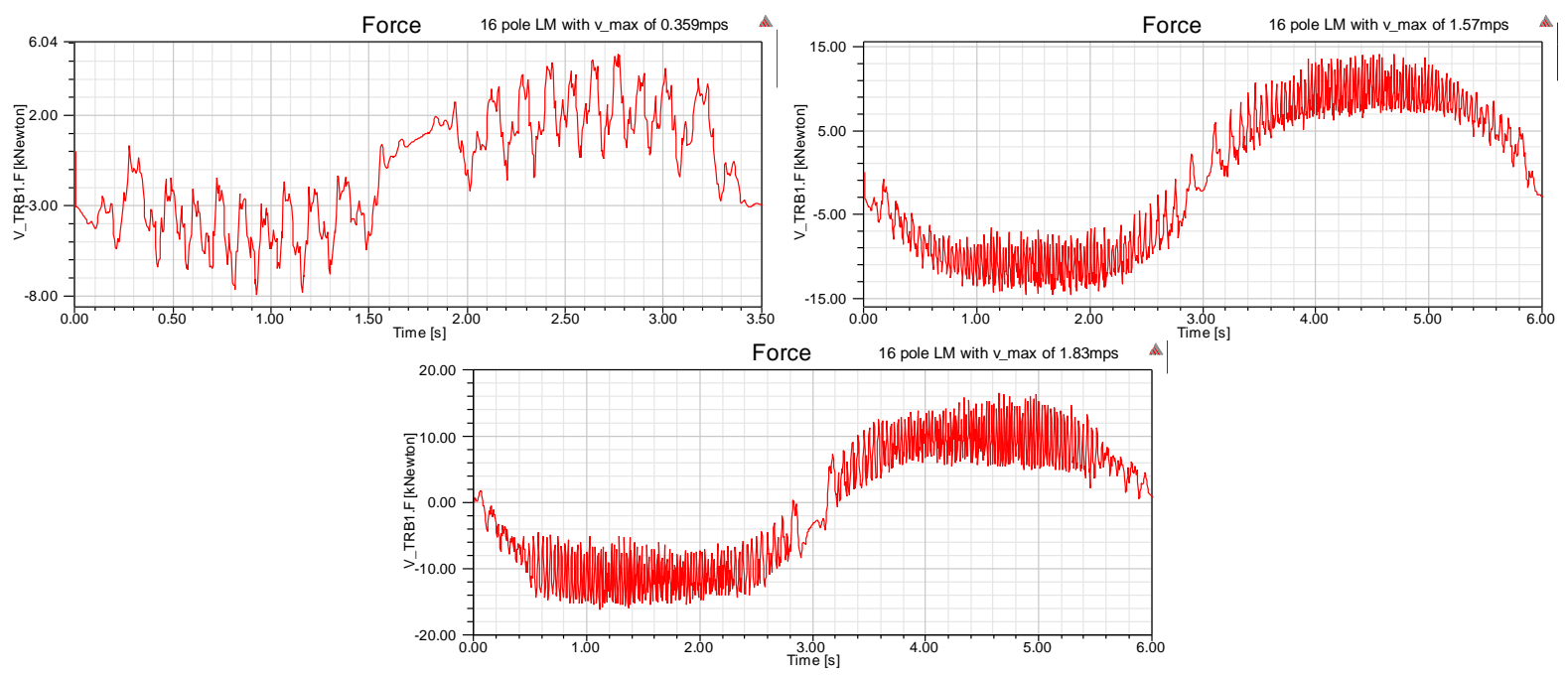

Figure 7: Performance of the 16-pole machine with variable speed, peak velocities of $0.359 \mathrm{~m} / \mathrm{s}, 1.57 \mathrm{~m} / \mathrm{s}$ and $1.83 \mathrm{~m} / \mathrm{s}$ respectively

The variable speed scenarios highlight the importance of having a high number of poles, especially at slow speed conditions. Had there been less poles the sinusoidal shape wave would have been lost due to the ripples in the force waveform. When the input velocity was representing slower, but the most common waves, a significant difference was clearly visible between the performances of the linear machines. 
The variable speed conditions have a direct effect on the generator's electrical outputs and on the force on the translator. Even though the direction of the force is continuously changing the machine is constantly in generating mode as the direction of the velocity is always opposing that of the force. Through this analysis one could also notice that the ripple in the force is lower for machines with a larger number of poles especially at slow speeds. The voltages induced at slow speeds in the 4-pole machine will have a larger harmonic content than that induced in the 16-pole machine. The frequency of the electrical outputs are higher as there are more complete cycles in one wave cycle in the 16-pole machine. This results in a smoother current flowing through the windings and a smoother output force from the 16-pole machine. These slow speeds represent the most common waves thus a smoother output will increase the efficiency of the linear machine design. Simulation result show that at $1.57 \mathrm{~m} / \mathrm{s}$ the- 16 pole machine would be $90 \%$ efficient.

For comparative reasons the double sided flat equivalent of the 16-pole machine was also simulated. The model resulted with a lower flux density in the air gap when compared to the tubular equivalent. A lower induced voltage was obtained and consequently a lower current for the same input velocity and resistive loading. Further details can be found in [6].

\section{Conclusions and Further Work}

Through this analysis it was therefore concluded that the 16-pole machine would be more suitable in the given application. The selected machine resulted to have a lower force ripple on the moving translator. Furthermore this machine design was also found to offer superior performance at slow speeds due to its higher output frequency. The higher the frequency the less rippled the output shall be. However it was not possible to lower the frequency further due to constraints related to the dimensioning of the mechanical system.

The analysis presented in this paper was mainly focused on the design of the electromechanical linear PM machine. Testing of the performance of the linear machine was carried out by operating the linear generator design together with a 3-phase resistive load bank. Further testing can also be carried out on the effects of non-linearities introduced when connecting such a machine to the rectification stage of a grid-connected inverter.

\section{Bibliography}

[1] Xuereb A, Spiteri Staines C, Mule`Stagno L, Sant T, Drago A, Azzopardi J, Gauci A “Analysis of the Potential of a Wave Energy Conversion System for Maltese Waters," SUSTAINABLE ENERGY 2013: THE ISE ANNUAL CONFERENCE, March 2013, Malta.

[2] “Ansys Maxwell,” Ansys, [Online]. Available: http://www.ansys.com/maxwell/. [Accessed 29 June 2014$].$

[3] “Ansys Simplorer," Ansys, [Online]. Available: http://www.ansys.com/Products/Simulation+Technology/Systems+\&+Multiphysics/Multiphysics+Enabled+Pr oducts/ANSYS+Simplorer. [Accessed 29 June 2014].

[4] B. Drew, R. Plummer and M. Sahinkaya, "A Review of Wave Energy Converter Technology," IMechE, vol. 223, p. 887, 2009.

[5] A. Falcão, "Wave energy utilization: A review of the technologies," Elsevier, vol. 14, no. 3, pp. 899-918, 2010.

[6] A. Xuereb, "Design of an Electrical Machine for a Wave Energy Converter," M.Sc. Thesis, University of Malta, 2013.

[7] P. J. Z. Gieras Jacek F, Linear Synchronous Motors: Transportation and Automation Systems, USA: CRC Press, 2011. 